\title{
La censura nella Francia settecentesca e la figura del colporteur tra realtà e finzione
}

\author{
Censure in eighteenth-century France and the figure of the colporteur \\ between reality and fiction
}

Luisa Messina

Università di Palermo, Italia

\begin{abstract}
Riassunto: La censura assume un'importanza fondamentale nella Francia settecentesca se si considera il vistoso aumento dei censori che passa da quarantuno all'inizio del secolo a settantotto alla vigilia della Rivoluzione. Cercando di raggirare i divieti imposti dalla censura, i colporteur ossia i venditori francesi ambulanti di libri eludano la sorveglianza nascondendo sotto il loro mantello sia scritti libertini che libelli politici, vale a dire i libri più amati e contestati dell'epoca, per poi venderli ai clienti più abbienti.
\end{abstract}

Parole chiave: censura, settecento, Francia, colporteur.

Abstract: Censorship is of fundamental importance in eighteenth-century France, a fact corroborated by the increase in the number of censors throughout the century: from forty-one in the early 1700 s to sixty-eight before the Revolution. In order to avoid the controls imposed by censure, colporteurs, that is to say clandestine book sellers, hid libertine works, political pamphlets, and the most controversial, yet loved books, in order to sell them to the richest clients.

Keywords: censure, eighteen century, France, colporteur.

In Francia la censura è ben organizzata in quanto ogni censore, che può essere anche scelto dall'autore, ha il compito di visionare un manoscritto che viene rigorosamente esaminato al fine di apporvi la consueta formula di approvazione. Prima della Rivoluzione non si può dare alle stampe un'opera senza che la premilitare censura e senza il privilegio del re'. A causa della disapprovazione della censura, Claude Crébillon è imprigionato nella prigione di Vincennes per aver pubblicato L'écumoire (1732) ed è, in seguito, esiliato da Parigi dopo l'apparizione de Le sopha (1742). L'approvazione del censore, tuttavia, non esonera da un'eventuale condanna dell'Università della Sorbona, del clero o del Parlamento locale (Alexandrian, 1989: 212). La censura settecentesca, inoltre, tiene sotto controllo le opere che osano mettere in discussione la religione, l'autorità pubblica e i costumi. L'attentato al re Luigi XV (1757), pertanto, provoca un inasprimento

\footnotetext{
1 Esiste, inoltre, una grande differenza tra il "privilegio" che, una volta approvato, è esclusivo tutelando l'autore e il "permesso" che non impedisce la concessione della stessa opera ad altre case editrici (Dalloz, 1836: 219).
} 
generale delle pene che vanno dalla pena di morte per gli autori, tipografi e colporteurs alla condanna in carcere per gli altri: i libri, inoltre, che mescolano irreligione e sregolatezza provocano una repressione più o meno severa (Wald Lasowski, 2011: 96-97)². La censura regia, dunque, considera la produzione libertina come un triplice affronto: un' offesa alla religione, all'autorità pubblica e all'integrità dei costumi (Bernier, 2001: 37). Non avendo l'approvazione della censura, i pamphlet politici, i libri deisti e atei nonché i romanzi erotici sono pubblicati clandestinamente sotto false indicazioni (Amsterdam, La Haye e Londra) o stampati direttamente in altri Paesi (Olanda, Inghilterra e Svizzera) dove non vige la censura (Alexandrian, 1989: 213). Le librerie straniere raggruppano i titoli di opere interdette nei cataloghi manoscritti clandestini che forniscono alle librerie francesi sotto la dicitura "livres philosophiques" che designa non tanto i libri nobili e prestigiosi dei Lumi, ma piuttosto un campo particolare del commercio riguardante le librerie ossia quello dell'illecito, del proibito, del clandestino, del perseguitato dalla polizia ma pur sempre fonte di possibile guadagno. Questi libri proibiti dalla diversa natura concernono l'erotismo, il trattato filosofico, il racconto fantastico, l'arringa e la cronaca dei costumi che sono accomunati dalla contestazione dell'ortodossia religiosa, politica, intellettuale, accademica accusando di ridicolo o coprendo di anatemi i valori tradizionali e i personaggi appartenenti alle sfere sociali più elevate (principi e re in particolar modo). In questi cataloghi proibiti due generi risultano, certamente, dominanti ossia le opere libertine (Dom Bougre, portier des chartreux, Margot la ravaudeuse. Thérèse philosophe in particolar modo) e i libelli di denuncia politica sotto forma di cronaca scandalosa (De Baecque e Mélonio, 1998: 64-65).

Uno degli scrittori libertini più sorvegliati dalla censura europea settecentesca è certamente François-Antoine Chevrier (1721-1762) i cui scritti contestatari sono perennemente controllati dalle autorità vigente visto che lo scrittore lorenese non si fa alcuno scrupolo a denigrare personalità della corte, fanciulle mondane, nobili lascivi, attrici del teatro dell'Opéra e commedianti, togati e finanzieri. Nonostante i rigidi divieti della censura Le colporteur, il romanzo più celebre di Chevrier, fa parte di quei libri proibiti (insieme alle opere di Rousseau e altre) che, pubblicati in Olanda, arrivano in Francia passando per Roven tramite Machuel e Besongue che ne detengono il monopolio tra gli anni 1763-1764 (Belin, 1913: 31)3. Chevrier deve affrontare le ostilità del ministro Choiseul, indiscusso protagonista della politica del tempo. Abile diplomatico, il primo ministro cerca di fronteggiare la potenza

\footnotetext{
2 È interessante, a tal riguardo, riportare alla luce la vicenda riguardante un noto venditore ambulante di libri, un tale Nicolas Gerlache, che suscita all'epoca particolarmente scalpore per la sua attività illecita e per le sue cattive compagnie che lo conducono in prigione. La sua esperienza, dunque, fornisce un'importante testimonianza delle condanne esemplari a cui possono andare incontro coloro che si oppongono all'Ancien Régime. Gerlacheri è un rilegatore di libri, colporteur, contrabbandiere, commerciante e direttore di un cabinet letterario a Metz che ama dipingersi come un vomo venuto dal nulla che si è fatto strada grazie al suo cervello. Dopo aver lavorato come conciatore, costui diventa rilegatore, venditore di libri finché le cattive compagnie lo conducono alla prigione di Bicêtre, destinata ai mascalzoni: il suo nome è, infatti, in cima alla lista dei "colporteurs et distributeurs de mauvais livres" contenuta in un rapporto di d'Hémery (Darnton, 1983: 114-115).

3 Chevrier viene costantemente tenuto sotto controllo dalla polizia. Un dei rapporti testimonia il tentativo di Chevrier di far arrivare seicento copie de Le colporteur a Parigi grazie alla complicità di un abate italiano: "Chevrier destine 600 exemplaires du Colporteur pour Paris. II le publie à ses dépens. II y a des anecdotes et des portraits. C'est un petit abbé italien, dont je ne sais pas le nom, qui est à Bruxelles et avec qui il est intimement lié, qui s'est chargé de faire passer cet ouvrage à Paris, et c'est à lui qui les 600 exemplaire seront adressées" (Richter, 1968: 266).
} 
britannica, che si sta alleando con la Prussia, stringendo un'alleanza sul continente con la casa d'Austria mediante il matrimonio del delfino di Francia, il futuro Luigi XVI, con l'arciduchessa Maria Antonietta (Capefigue, 1843: 5). A seguito dello scandalo suscitato in Francia al momento della pubblicazione de Le colporteur, il ministro Choiseul inizialmente emette una lettre de cachet e successivamente ordina l'immediata incarcerazione alla Bastiglia e l'arresto di Chevrier, che non è affatto a Parigi come crede erroneamente il ministro ${ }^{4}$. Choiseul, allora, rende noto che il re ha ordinato di arrestare Chevrier per farlo imprigionare alla Bastiglia ${ }^{5}$. Per uno scrittore la detenzione alla Bastiglia rappresenta, comunque, l'anticamera della celebrità (Stroev, 1997: 62). Dopo aver raccolto numerose informazioni riguardanti la condotta di Chevrier, un sorvegliante consegna al tenente di polizia M. de Sartines ${ }^{6}$ il suo rapporto nel quale Chevrier è dipinto come un scrittore pericoloso nonché nemico della Francia meritevole di trascorrere il resto dei suoi giorni alla Bastiglia non solo per aver pubblicato insolentemente le sue opere, ma anche per aver lavorato con una spia prussiana ${ }^{7}$.

\footnotetext{
4 Le lettres de cachet sono lettere emanate dal sovrano che contengono ordini, comandi o avvisi del re. II loro obiettivo è sovente quello di mandare qualcuno in esilio o per prelevarlo e farlo chiudere in prigione (come nel caso di Chevrier). Le lettres de cachet sono un segno tangibile del dispotismo monarchico e, pertanto, sono abolite dai rivoluzionari.
}

II 3 settembre del 1761 Choiseul scrive una lettera all'ambasciatore austriaco in Francia in cui chiede di intercedere presso il governatore dei Paesi Bassi e il conte Cobenzl per bloccare la pubblicazione del Gazetin di Chevrier in nome dell'amicizia e dell'alleanza tra le due grandi nazioni e per ottenerne l'estradizione, giudicato indegno di ogni protezione: "Le sieur Chevrier, mauvais sujet à tous égards, et que des affaires peu honorables pour lui ont forcé de sortir de France, fait imprimer publiquement à Bruxelles une feuille intitulée 'Gazetin' dans laquelle il a l'effronterie d'insinuer des faits qui sont d'une fantaisie évidente et des réflexions d'une insolence extrême. Son Altesse Royale Monseigneur le Gouverneur des Pay-Bas et Monsieur le comte de Cobenzl sont trop éclairés et trop équitables pour ne pas sentir combien une pareille licence est contraire à l'ordre public et peu convenable aux liens d'amitié et d'alliance qui unissent nos maîtres respectifs. II serait même fort à désirer qu'en supprimant le 'Gazetin' scandaleux dont il s'agit, on voulût bien nous accorder l'extradition de l'auteur, qui, en manquant à ses devoirs de sujet du roi, s'est rendu indigne de toute protection, surtout de la part des alliés de sa Majesté. II est certain qu'en pareille circonstance sa Majesté ne ferait nulle difficulté de différer à la réquisition de sa Majesté impériale la reine apostolique" (Richter, 1968: 255).

La lettera è stata, certamente, ben accolta se si considera che il 7 settembre del 1761 Choiseul comunica la soppressione del giornale sebbene riconosca le difficoltà nell'estradizione: "On nous accorde, Monseigneur, la suppression entière de cette insolente feuille. Le ministre m'a assuré qu'elle ne paraîtra plus, mais l'extradition de l'auteur souffre les plus grandes difficultés dans le Brabant, dont les privilèges y mettent obstacle" (Richter, $1968: 256)$.

5 "Je reçois la lettre que vous m'avait fait l'honneur de m'écrire hier avec les ordres du Roi qui y étaient joints pour arrêter et conduire à la Bastille le $\mathrm{S}^{r}$ Chevrier arrivé depuis quelque temps de Bruxelles et dont on ne sait la demeure et dans l'instant j'ai chargé un Inspecteur de police de faire toutes les recherches imaginables pour tacher de découvrir cet homme" (L. J. F. Ravaisson-Mollien, 1914: 233).

${ }^{6}$ M. de Sartines, spagnolo di nascita, diventa il prefetto della polizia parigina nel 1759 e porta il sistema di spionaggio poliziesco ai gradi più alti di perfezione. Chiamato il "Grande Inquisitore" della polizia, egli giustifica tutti i mezzi e metodi perché vuole conoscere tutto e vedere tutto. M. de Sartines, non a caso, risulta uno degli uomini più odiati nei romanzi di Sade (Bloch, 2011: 100).

7 "Par une relation que j'ai en Hollande pour connaître les mauvais sujets de Paris qui se réfugient quand ils ont fait des friponneries ici, ou connaître les étrangers suspects qui viennent à Paris, je vois souvent sur la scène deux Français [Gaubier de Barrault et Chevrier] qui sont à La Haye, mauvais sujets, esprits satiriques, ennemis déclarés de leur patrie, faisant métiers d'auteurs, qui sont en relation avec nos frondeurs de Paris, qui leur envoient des vers, des anecdotes contre tout ce qu'il y a de plus respectable en France, et qu'ils font imprimer et courir avec la plus grande insolence; ces deux hommes mériteraient de passer leurs jours à la B. [astille], étant des chenilles et des pestes publiques. [...] L'autre se nomme Chevrier, contre lequel M. de Choiseul a fait expédier un ordre du Roi du 1 er 
Il protagonista del romanzo di Chevrier ci permette di comprendere le caratteristiche salienti di questa figura posta ai margini della società francese. Monsieur Brochure, definito come il rivenditore di libri meglio fornito e più scandaloso del regno (Chevrier, 1993: 756) è solo apparentemente un venditore ambulante di libri. Tra gli anni della Reggenza e la metà del Settecento i rapporti di polizia hanno, infatti, documentato un intenso traffico di manoscritti condannati dalla censura ma venduti di nascosto grazie ai copisti e ai colporteurs clandestini come certi Bonnet, Lecoulteux, ai quali sono sequestrati testi spinoziani nel 1729, e Mathieu o Morléon. All'estero i colporteurs che comunicano le novità si assumano delle arie: numerosi venditori olandesi si prendono gioco persino degli vomini politici. Chevrier, a tal riguardo, si burla di "ces négociateurs subalternes que l'on nomme, en termes de l'art, frippiers de nouvelles, ou furets d'anti-chambres" (Chevrier, 1762: 42). La circolazione clandestina dei manoscritti, comunque, apre la strada alla propaganda antireligiosa e politica dei philosophe (P. Casini, 1973: 218). I venditori, inoltre, non si accontentano di consegnare i libri ai portieri ma sono, al contrario, accolti nelle dimore nobiliari mostrando la loro mercanzia ai loro protettori: essi sono, dunque, fornitori abituali della buona società al pari dei gioiellieri o venditori di stoffe, ragion per cui il loro mestiere li tiene continuamente in contatto con persone distinte consentendo loro di conoscere gli scandali della corte e della città (Belin, 1913: 9394) ${ }^{8}$. F. Gevrey ha, infatti, messo in rilievo che i numerosi venditori di libri accedevano ai boudoirs e alle toilettes delle dame: i colporteurs hanno così modo di poter proporre scritti libertini o semplicemente presentare delle storie e commentarle. Sono state, inoltre, rinvenute le origini letterarie della figura del colporteur risalenti al XVII secolo: romanzi e novelle secentesche hanno, infatti, testimoniato la presenza di questi venditori ambulanti i quali trasportano piccoli oggetti (gioielli e immagini) 0 , talvolta, si travestono per poter raggiungere le donne amate (Gevrey, 2006: 121122). Se i venditori ambulanti vengono scoperti, talvolta subiscono pene moderate come la gogna, la messa al bando temporanea dalla città o dal libraio di riferimento, l'incarcerazione alla Bastiglia. La detenzione alla Bastiglia, tradizionalmente riservata ai nobili che vengono trattati come pensionati del re, implica un certo coinvolgimento nell'ambito letterario: anche un venditore ambulante si sente molto considerato se viene rinchiuso alla Bastiglia invece che alle prigioni la Force e Châtelet, riservati solitamente ai volgari delinquenti (Alexandrian, 1989: 215). P. Wald Lasoswi ha definito il colporteur un agente attivo di libri proibiti che, sebbene continuamente minacciato di multa, infamia, imprigionamento o galera (a seconda della natura dei libri messi in circolazione), diffonde brochure sfuggendo la polizia: il critico riconosce a Chevrier il merito di aver riportato alla ribalta tale figura con la pubblicazione dell'omonimo romanzo (Wald Lasowski, 201 1: 113). R. Trousson ha messo in rilievo che tale figura è tipica del contesto storico-

novembre 1761, pour le mettre à la B., avec ses papiers, le croyant à Paris. [...] Et Chevrier, en 1754, s'étant rendu suspect parce qu'il fournissait à Morand, auteur, qui était espion du roi de Prusse, toutes les nouvelles de Paris et de la Cour, que Morand envoyait à ce prince. M. Berryer envoya faire une perquisition chez lui, où l'on trouva beaucoup de minutes de nouvelles qui furent saisies, néanmoins il ne fut pas arrêté" (L. J. F. Ravaisson-Mollien, 1881: 463-464).

8 I libri condannati dalla censura, paradossalmente, raggiungono gli appartamenti lussuosi di Versailles. Ad esempio, Le portier de Chartreux, il cui successo è stato certamente incrementato dalla curiosità avida del pubblico, arriva nelle mani di Maria Adelaide, la quarta figlia di Luigi XV, sorpresa di leggerlo. Questo libro è d'altronde circolato clandestinamente sotto il mantello per tutto il Settecento (Andrei, 2006: 71). 
sociale del Settecento: il venditore ambulante di libri settecentesco, infatti, si occupa della diffusione di opere proibite filosofiche e scritti libertini che vengono divulgati e venduti ai clienti attraverso tre espedienti: i venditori si recano personalmente all'interno di luoghi pubblici (caffè, teatri, strade, passeggio), ma anche all'aria aperta (nel corso di una passeggiata) o, talvolta, nelle dimore nobiliari tramite la vendita a domicilio (Trousson, 1993: 745). È, certamente, il caso del colporteur, protagonista dell'omonimo romanzo di Chevrier, che viene chiamato a interloquire con un'intrigante nobildonna che lo esorta a renderle noti piccanti aneddoti tenuti segreti. F. Gevrey ha, a tal proposito, affermato che il personaggio di Monsieur Brochure era indubbiamente l'autore delle storie galanti udite dalla marchesa e dal cavaliere: la studiosa si è, quindi, chiesta se Chevrier abbia scelto l'assimilazione dell'autore al venditore per svalutare il ruolo dello scrittore o per nascondere il veleno di queste storie narrate (Gevrey, 2006: 122). Oltre a passare da una casa nobiliare ad un'altra raccontando le sue storie, il colporteur di Chevrier ha svelato la sua reale professione di spia al servizio della polizia'. L'vomo, successivamente, afferma di essere il colporteur che rifornisce Versailles da dieci anni. F. Gevrey ha individuato le caratteristiche salienti del personaggio del colporteur che è stato più volte etichettato perruque (uomo dalle idee antiche) dal cavaliere, ha dato prova di una certa eloquenza e attacca sia alcuni scrittori (Palissot, autore de Philosophe) che attrici e donne mantenute (Gevrey, 2006: 131). S. Aragon ha brevemente presentato il romanzo mettendo in luce, da una parte, la critica al ridicolo e ai vizi dominanti la società e, dall'altra, i giudizi sulle opere contemporanee espressi dai tre personaggi del romanzo ${ }^{10}$.

L'analisi della figura del colporteur che emerge dall'omonimo romanzo di Chevrier è necessaria per comprendere il clima di censura che domina la Francia settecentesca alla vigilia della Rivoluzione francese ${ }^{11}$. La censura, infatti, risulta un'organizzazione ben regolata nella quale lavorano in sinergia censori ufficiali e spie in incognito in grado di bloccare l'apparizione delle opere illuministe (la pubblicazione dell'Enciclopedia è bloccata svariate volte) tanto gli scritti di scrittori considerati secondari (come Crébillon e Chevrier) nel panorama letterario francese. Nonostante il controllo coercitivo esercitato dalla censura, alcuni colporteur

\footnotetext{
9 "Je ne suis point colporteur, et cette médaille que vous me voyez n'est pas qu'un passeport que la police me donne pour aller, en portant des livres sous le manteau, épier les anecdotes scandaleuses, et les aventures galantes dont je compose le soir un petit memoria que je porte au bureau" (Chevrier, 1993: 760).

10 "François-Antoine Chevrier a publié en 1753, les Mémoires d'une femme honnête écrits par ellemême (publié à Londres), avant d'entrer en politique. II déclare dans l'avertissement du Colporteur revenir à l'écriture pour déclarer la guerre aux ridicules et aux vices. Son héroïne, la marquise de Sarmé, est une libertine qui s'intéresse à la littérature l'âge venant et ses appâts déclinant. Elle reçoit le chevalier, un ancien amant, lorsque le colporteur vient lui présenter ses nouveautés. Le roman raconte la découverte à trois des dernières ouvrage publiés sous le manteau" (Aragon, 2003: 317).

11 L'abolizione della censura, dunque, risulta una delle priorità avvertite dai rivoluzionari. Tra il 14 luglio 1789 e il 10 agosto 1792 la libertà di stampa è pressoché illimitata a dispetto dei tentativi di regolamentazione, della proposta di legge di Sieyès e del progetto Thouret. In seguito alla soppressione della censura e alla proclamazione della libertà di stampa, compaiono infatti numerosi romanzi che presentano ora descrizioni dettagliate di amplessi amorosi ora il libertinaggio più perverso apertamente ostentato dalle prostitute come nel romanzo sadiano Justine ou les malheurs de la vertu (1791). Nel 1810 viene infine instaurato un nuovo clima di paura e sospetto, incoraggiato d'altronde dal ripristino della censura e dalla stesura del Codice penale, che mira a scoraggiare la circolazione di opere clandestine.
} 
coraggiosi sfidano le autorità vigenti vedendo clandestinamente i libri considerati empi in quanto affrontano scottanti questioni inerenti la politica o la sessualità. II personaggio di Monsieur Brochure, frutto della penna di Chevrier, ben sintetizza le caratteristiche di una delle figure sociali poste ai margini della società.

\section{Bibliografia}

ALEXANDRIAN, S. (1989) : Histoire de la littérature érotique. Paris : Seghers.

ANDREI, C. (2006): Romans libertins du XVIIle siècle. Configurations narratives. Bucarest : Editura Didactica si pedagogica.

ARAGON, S. (2003) : Des liseuses en péril. Paris : Champion.

BELIN, J.-P. (1913): Le commerce des livres prohibés à Paris de 1750 à 1789. Paris: Belin frères.

BERNIER, M.-A. (2001) : Libertinage et figures du savoir. Rhétorique et roman libertin dans la France des Lumières. Québec-Paris: Presses de I'Université LavalL'Harmattan, Collection République des lettres.

BLOCH, I. (2011): Marquis de Sade's 120 Days of Sodom or the School for Libertinage and the Sex Life of French Age of Debauchery [1934]. Whitefish: Kessinger.

CAPEFIGUE, J.-B. H. R. (1843) : Louis XV et la société du dix-huitième siècle. Bruxelles: Méline, Cans et Compagnie.

CASINI, P. (1973): Introduzione all'Illuminismo. Da Newton a Rousseau. Bari: Laterza.

CHEVRIER, F.-A. (1993[1761]) : Le colporteur. R. TROUSSON (éd.) : Romans libertins du $X V I I I$ siècle. Paris : Laffont.

- (1762) : Les Amusements des dames de $B^{* * *}$. Histoire honnête et presque édifiante, composée par feu le chevalier de $\mathrm{Ch}^{* * * * *}$ et publiée par l'auteur du Colporteur. Roven : Pierre Le Vrai.

DALLOZ, D. (1836): Jurisprudence générale du royaume. Recueil périodique et critique de législation, de doctrine et de jurisprudence. Paris: Imprimerie de Madame Veuve Poussin.

DARNTON, R. (1983) : Bohème littéraire et révolution. Paris : Sevil.

DE BAECQUE, A. et F. MELONIO (1998) : Histoire culturelle de la France. Paris : Seuil.

GEVREY, F. (2007) : "L'auteur colporteur: une représentation de l'écrivain au XVIIIe siècle ॥. Travaux de littérature, $\mathrm{n}^{\circ}$ 20, pp. 121-133.

RAVAISSON-MOLLIEN, L.-J.-F. (1881): Archives de la Bastille, Volume XII. Paris: A. Durand et Pedone-Lauriel.

- (1914) : «Notes et Rapports de Police. Extraits des Archives de la Bastille ». F.-A. CHEVRIER : Le colporteur, édition établie sous la direction d'A. Van Bever. Paris: Bibliothèque des curieux, pp. 231-244.

RICHTER, E. (1968): Chevrier. Ein Satiriker im Kampf mit dem ancien Régime. Thèse Berlin deutsche Akademie der Wissenschaften.

STROEV, A. (1997) : Les aventuriers des Lumières. Paris : P.U.F.

TROUSSON, R. (1993) : "Introduction au Colporteur». R. TROUSSON (éd.) : Romans libertins du XVIII siècle. Paris : Laffont, pp. 741-750.

WALD LASOWSKI, P. (2011) : Dictionnaire libertin. Paris : Gallimard. 